\title{
Duration of Chemotherapies in Phase III Studies on Metastatic Colorectal Cancer Treated With a Continuous Approach: A Seven-Year Survey
}

Alessandro Ottaiano ${ }^{1 *}$, Maurizio Capuozzo ${ }^{2}$, Guglielmo Nasti ${ }^{1}$, Anna Nappi ${ }^{1}$, Chiara De Divitiis ${ }^{1}$, Monica Capozzi ${ }^{3}$, Salvatore Tafuto ${ }^{1}$, Piera Maiolino ${ }^{3}$ and Vincenzo Rosario laffaioli ${ }^{1}$

${ }^{1}$ Department of Abdominal Oncology, National Cancer Institute, "G. Pascale" foundation, via M. Semmola, 80131, Naples, Italy

${ }^{2}$ Department of Pharmacy at the Local Sanitary Agency (LSA) Naples 3 South, Herculaneum, Naples, Italy

${ }^{3}$ Department of Pharmacy, National Cancer Institute, “G. Pascale" foundation, via M. Semmola, 80131, Naples, Italy

\begin{abstract}
In last years, many phase III studies have addressed the role of chemotherapy or chemo/biotherapy in metastatic colorectal cancer (mCRC); most of these studies planned their treatment to be continued until disease progression or intolerable toxicity with the aim to obtain a long-term control of the cancer. However, the real duration of this approach might be significantly lower than planned. We made a survey to describe the real duration of chemotherapy in published phase III trials from January 2008 to December 2014. Twenty relevant publications were selected for a total of 48 treatment arms and of 24.475 patients. Median duration of chemotherapy in first-line studies ranged from 4,8 to 7,8 months; in second line from 2,4 to 5,2 months. Most common reasons of discontinuation were: progressive disease (PD), adverse events (AE) and patient request (PR). From $11.0 \%$ to $45.0 \%$ of patients discontinue treatment for toxicity or their request independently from the efficacy. PR was the third cause ranging from $4,6 \%$ to $26,0 \%$ of patients; in some studies, it overcame the AE-related withdrawals. Causes of PR for therapy discontinuation should be explored and analyzed to reduce the proportion of withdrawals in phase III studies.
\end{abstract}

Keywords: Colorectal cancer; Phase III studies; Toxicity; Chemotherapy; Survey

\section{Introduction}

The recent introduction of biologic therapies (cetuximab, bevacizumab, panitumumab) in combination with chemotherapy (fluoropirimidines, oxaliplatin, irinotecan), has extended median overall survival of patients with $\mathrm{mCRC}$ to two years and beyond [1]. Survival of 30 months and beyond can be reached in molecularly defined subpopulations [2]. As with other low-proliferating tumors (i.e. breast cancer), a sequential and continuous treatment approach using all active agents can obtain a long-term control of the disease and prolong survival [3]. Thus, for many patients, these developments have changed the therapeutic perspective of $\mathrm{mCRC}$ from an acute to a chronic condition. In last years, many randomized phase III studies have addressed the role of chemotherapy or chemo/biotherapy in mCRC; most of these studies planned their treatment to be continued until disease progression or intolerable toxicity. However, the real duration of this approach might be significantly lower than planned. How many patients are able to receive the planned treatment until progression or toxicity? Which is the burden of patient-related decisions in early discontinuation of therapies? We made a literature survey focused on this topic, to describe what is reported in published phase III studies.

\section{Methods}

We searched PubMed to describe the duration of chemotherapy in mCRC treated with chemotherapy planned until progressive disease or unacceptable toxicity (continuous approach). The upper data limit of January 2008 and the lower date limit of December 2013 were applied. The search strategy combined the following terms: (colorectal OR colon OR rectum OR colorectum OR) AND (cancer ${ }^{\star}$ OR carcinoma ${ }^{\star}$ OR neoplasm ${ }^{\star}$ OR tumor ${ }^{\star}$ ). Thereafter, the search was limited to clinical, phase III, human studies. Ancillary or economic evaluations (as primary objective) subgroup or prognostic or interim/updated long-term analyses were excluded. Given the importance of $\mathrm{AE}$ in determining duration of treatments, phase III studies were selected using a 16-point $\mathrm{AE}$ reporting quality score (AERQS) based on the
2004 CONSORT extension [4]. Only studies with AERQS score $>10$ were selected. Other selection criteria were: prospective studies and first or second-line therapies. Selection was limited to publications in English language. The collected informations are reported in Table 1. When the information on treatment discontinuation was not reported in the text, it was extracted from tables and/or consort diagram. In antiEGFR studies, when subgroup analyses were available, the description of data was restricted to KRAS WT-tumors.

Sixty-seven papers were selected. Reasons for exclusion were as follow: 15, AERQS $\leq 10 ; 7$, ancillary; 3 , economic evaluations; 6 , subgroup analyses; 4 , prognostic; 5 , interim or updated long-term analyses; 7 , more than one reason.

\section{Results}

Twenty relevant publications were selected from 2008 to 2014 for a total of 48 treatment arms and of 24.475 patients. Fifteen studies reported on first-line therapy; five on second-line. Duration of chemotherapy in first-line studies ranged from 4.8 to 7.8 months. In second line from 2.4 to 5.2 months. The three most common reasons of chemotherapy discontinuation were: progressive disease $(\mathrm{PD})$, adverse events (AE) and patient request (PR). $\mathrm{PD}$ and $\mathrm{AE}$ were the major reasons of discontinuation in both first- and second-line

*Corresponding author: Alessandro Ottaiano, M.D., Department of Abdomina Oncology, National Cancer Institute, "G. Pascale" foundation, via M. Semmola 80131, Naples, Italy, Tel: +39 081 5903367; Fax: +39 081 3606259; E-mail: ale.otto@libero.it

Received June 04, 2015; Accepted July 06, 2015; Published July 10, 2015

Citation: Ottaiano A, Capuozzo M, Nasti G, Nappi A, De Divitiis C (2015) Duration of Chemotherapies in Phase III Studies on Metastatic Colorectal Cancer Treated With a Continuous Approach: A Seven-Year Survey. J Cancer Sci Ther 7: 202-205. doi:10.4172/1948-5956.1000351

Copyright: (c) 2015 Ottaiano A, et al. This is an open-access article distributed under the terms of the Creative Commons Attribution License, which permits unrestricted use, distribution, and reproduction in any medium, provided the original author and source are credited. 
Citation: Ottaiano A, Capuozzo M, Nasti G, Nappi A, De Divitiis C (2015) Duration of Chemotherapies in Phase III Studies on Metastatic Colorectal Cancer Treated With a Continuous Approach: A Seven-Year Survey. J Cancer Sci Ther 7: 202-205. doi:10.4172/1948-5956.1000351

\begin{tabular}{|c|c|c|c|c|c|c|c|c|c|c|c|}
\hline \multirow[b]{2}{*}{ First Author } & \multirow[b]{2}{*}{ Year } & \multirow[b]{2}{*}{$\begin{array}{l}\text { No. of } \\
\text { patients }\end{array}$} & \multirow[b]{2}{*}{$\begin{array}{l}\text { Line of } \\
\text { treatment }\end{array}$} & \multirow[b]{2}{*}{ Arms } & \multicolumn{2}{|c|}{$\begin{array}{l}\text { Median duration of } \\
\text { chemotherapy }\end{array}$} & \multicolumn{2}{|c|}{$\begin{array}{l}\text { Median Outcome } \\
\text { (months) }\end{array}$} & \multicolumn{3}{|c|}{$\begin{array}{l}\text { Fist three most common reasons of } \\
\text { discontinuation (\%of patients) }\end{array}$} \\
\hline & & & & & $\begin{array}{l}\text { No. of } \\
\text { cycles }\end{array}$ & Months & PFS & OS & & & \\
\hline \multirow[t]{2}{*}{ Rothenberg } & 2008 & 627 & $2^{\circ}$ & Folfox & 8,5 & NR & 4,8 & 12,5 & PD (46.0) & $\mathrm{AE}(14.0)$ & NR \\
\hline & & & & Xelox & 6 & NR & 4,7 & 11,9 & PD (38.0) & $\mathrm{AE}(21.0)$ & NR \\
\hline \multirow[t]{4}{*}{ Cassidy } & 2008 & 2034 & $1^{\circ}$ & $\begin{array}{l}\text { Folfox (or Folfox/ } \\
\text { Placebo) }\end{array}$ & 10 & NR & 7,7 & \multirow{2}{*}{$\begin{array}{c}\text { Pooled } \\
\text { folfox } \\
\text { arms: } 19,6\end{array}$} & NR & NR & $\mathrm{AE}(25.0)$ \\
\hline & & & & Folfox/Bev & 12 & NR & 9,4 & & NR & NR & $A E(30.0)$ \\
\hline & & & & $\begin{array}{l}\text { Xelox (or Xelox/ } \\
\text { Placebo) }\end{array}$ & 7 & NR & 7,3 & \multirow{2}{*}{\begin{tabular}{|c|} 
Pooled \\
xelox \\
arms: 19,8
\end{tabular}} & NR & NR & $\mathrm{AE}(26.0)$ \\
\hline & & & & Xelox/Bev & 8 & NR & 9,3 & & NR & NR & $\mathrm{AE}(31.0)$ \\
\hline \multirow[t]{4}{*}{ Saltz } & 2008 & 1401 & $1^{\circ}$ & Folfox/Bev & \multirow[t]{2}{*}{ NR } & \multirow[t]{2}{*}{6,3} & \multirow[t]{2}{*}{8,0} & \multirow[t]{2}{*}{19,9} & \multirow[t]{2}{*}{ PD (29.0) } & \multirow[t]{2}{*}{$\mathrm{AE}(30.0)$} & \multirow[t]{2}{*}{ PR (9.0) } \\
\hline & & & & Xelox/Bev & & & & & & & \\
\hline & & & & Folfox/Placebo & NR & 5,8 & 9,4 & 21,3 & PD (47.0) & $\mathrm{AE}(21.0)$ & PR (8.0) \\
\hline & & & & Xelox/Placebo & & & & & & & \\
\hline Sobrero & 2008 & 1298 & $2^{\circ}$ & Cet/iri & NR & 3,5 & 4,0 & 10,7 & PD (67.5) & PR (7.7) & $\mathrm{AE}(6.5)$ \\
\hline & & & & Iri & NR & 2,4 & 2,6 & 10,0 & PD (68.0) & PR (6.6) & $\mathrm{AE}(4.8)$ \\
\hline Haller & 2008 & 628 & $2^{\circ}$ & Irox & 6 & NR & 5,3 & 13,4 & PD (54.0) & PR (16.0) & $\mathrm{AE}(13.0)$ \\
\hline & & & & Iri & 4 & NR & 2,8 & 11,1 & PD (66.0) & $\mathrm{AE}(13.0)$ & PR (10.0) \\
\hline Cunningham & 2009 & 725 & $1^{\circ}$ & Oxa/FU CIV Arm A & 10 (information on & NR & A: 7,9 & A: 15,9 & PD (43.0) & $\mathrm{AE}(17.1)$ & PR (13.5) \\
\hline & & & & Folfox4 Arm A & & NR & & & & & \\
\hline & & & & FU CIV Arm B & & NR & B: 5,9 & B: 15,2 & PD (61.4) & PR (11.5) & $\mathrm{AE}(4.7)$ \\
\hline & & & & LV5FU2 Arm B & & NR & & & & & \\
\hline Van Cutsem & 2009 & 1217 & $1^{\circ}$ & Folfiri & NR & 6 & 8,0 & 18,8 & & NR & \\
\hline & & & & Folfiri/Cet & NR & 6 & 8,9 & 19,9 & & & \\
\hline Tol & 2009 & 755 & $1^{\circ}$ & Xelox/Bev & 10 & 7 & 10,7 & 20,3 & PD (54.0) & $\mathrm{AE}(25.9)$ & PR (6.1) \\
\hline & & & & Xelox/Cet & 9 & 6 & 9,4 & 19,4 & PD (48.5) & $\mathrm{AE}(29.6)$ & PR (7.5) \\
\hline Hecht & 2009 & 1053 & $1^{\circ}$ & Folfox/PanBev & NR & NR & 9,8 & 20,7 & PD (29.0) & $\mathrm{AE}(22.0)$ & PR (16.0) \\
\hline & & & & Folfox/Bev & NR & NR & 11,5 & 24,5 & PD (26.0) & $\mathrm{AE}(24.0)$ & PR (21.0) \\
\hline & & & & Folfiri/PanBev & NR & NR & 10,0 & $\begin{array}{c}\text { Not } \\
\text { Estimable }\end{array}$ & PD (35.0) & $\mathrm{AE}(17.0)$ & PR (15.0) \\
\hline & & & & Folfiri/Bev & NR & NR & 12,5 & 19,8 & PD (27.0) & PR (26.0) & $\mathrm{AE}(6.0)$ \\
\hline Douillard & 2010 & 1183 & $1^{\circ}$ & Folfox/Pan & $\begin{array}{l}\text { FU 12; } \\
\text { Oxa 11; } \\
\text { Pan } 10\end{array}$ & NR & 9,6 & 23,9 & $\%$ or abs & $\begin{array}{l}\mathrm{PD}, \mathrm{AE}, \mathrm{PR} \\
\text { olute number we } \\
\text { specified }\end{array}$ & ere not \\
\hline & & & & Folfox & $\begin{array}{l}\text { FU 12; } \\
\text { Oxa } 11\end{array}$ & NR & 8,0 & 19,7 & & & \\
\hline Peeters & 2010 & 1186 & $2^{\circ}$ & Folfiri/Pan & NR & NR & 5,9 & 14,5 & & $P D, A E, P R$ & \\
\hline & & & & Folfiri & NR & NR & 3,9 & 12,5 & $\%$ or abs & $\begin{array}{l}\text { olute number we } \\
\text { specified }\end{array}$ & ere not \\
\hline Maughan & 2011 & 2445 & $1^{\circ}$ & Folfox or Xelox & NR & 7,2 & 8,6 & 17,9 & & NR & \\
\hline & & & & $\begin{array}{l}\text { Folfox/Cet or Xelox/ } \\
\text { Cet }\end{array}$ & NR & 7,2 & 8,6 & 17,0 & & & \\
\hline Hecht & 2011 & 1168 & $1^{\circ}$ & Folfox/Vatalanib & NR & 6,4 & 7,7 & 21,4 & PD (51.2) & $\mathrm{AE}(22.3)$ & PR (14.7) \\
\hline & & & & Folfox/Placebo & NR & 7,8 & 7,6 & 20,5 & PD (66.5) & $\mathrm{AE}(12.1)$ & PR (10.1) \\
\hline Schmoll & 2012 & 1805 & $1^{\circ}$ & Folfox/Ced & 10 & $N R$ & 9,9 & 22,8 & PD (43.2) & PR (20.7) & $\mathrm{AE}$ (19.2) \\
\hline & & & & Folfox/Bev & 12 & NR & 10,3 & 21,4 & PD (42.7) & PR (18.6) & $\mathrm{AE}(18.4)$ \\
\hline Madi & 2012 & 2445 & $1^{\circ}$ & Xelox vs Folfox & NR & NR & 7,4 vs 8,8 & $\begin{array}{c}15,4 \text { vs } \\
14,9\end{array}$ & & NR & \\
\hline & & & & $\begin{array}{c}\text { Xelox/Cet vs Folfox/ } \\
\text { Cet }\end{array}$ & NR & NR & 7,4 vs 8,5 & $\begin{array}{c}15,0 \text { vs } \\
14,9\end{array}$ & & & \\
\hline Van Cutsem & 2012 & 1401 & $2^{\circ}$ & Folfiri/Aflibercept vs & 9 & 5,25 & 6,9 & 13,5 & PD (50.4) & $\mathrm{AE}(26.9)$ & PR (13.7) \\
\hline & & & & Folfiri/Placebo & 8 & 4,52 & 4,7 & 12,0 & PD (72.2) & $\mathrm{AE}(12.2)$ & PR (7.4) \\
\hline Hoff & 2013 & 1076 & $1^{\circ}$ & $\begin{array}{l}\text { Folfox/Xelox/Ced } \\
20 \mathrm{mg}\end{array}$ & $9 / 10 / 6$ & NR & 8,6 & 19,7 & PD (56.7) & $\mathrm{AE}(22.5)$ & PR (10.1) \\
\hline & & & & Folfox/Xelox/Placebo & $11 / 11 / 7$ & NR & 8,3 & 18,9 & PD (69.8) & $\mathrm{AE}(13.9)$ & PR (7.8) \\
\hline Carrato & 2013 & 768 & $1^{\circ}$ & Folfiri/Sunitinib & NR & NR & 7,8 & 20,3 & PD (42.4) & $\mathrm{AE}(24.0)$ & PR (4.6) \\
\hline & & & & Folfiri/Placebo & NR & NR & 8,4 & 19,8 & PD (39.0) & $\mathrm{AE}(11.2)$ & PR (5.8) \\
\hline Loupakis & 2014 & 508 & $1^{\circ}$ & Folfiri/Bev & 12 & NR & 9,7 & 31,0 & PD (30.3) & $\begin{array}{c}\text { "Other } \\
\text { reasons" (3.9) }\end{array}$ & $\mathrm{AE}(3.5)$ \\
\hline
\end{tabular}


Citation: Ottaiano A, Capuozzo M, Nasti G, Nappi A, De Divitiis C (2015) Duration of Chemotherapies in Phase III Studies on Metastatic Colorectal Cancer Treated With a Continuous Approach: A Seven-Year Survey. J Cancer Sci Ther 7: 202-205. doi:10.4172/1948-5956.1000351

\begin{tabular}{|c|c|c|c|c|c|c|c|c|c|c|c|}
\hline & & & & Folofoxiri/Bev & 11 & NR & 12,1 & 25,8 & PD (13.6) & $\mathrm{AE}(8.4)$ & $\begin{array}{c}\text { Deaths } \\
\text { (0.2\%: } 6 \\
\text { pts) }\end{array}$ \\
\hline Heinemann & 2014 & 752 & $1^{\circ}$ & Folfiri/Cet & 10 & 4,8 & 10,0 & 28,7 & $A E(15.0)$ & \multicolumn{2}{|c|}{ "Other reasons" (9.4) } \\
\hline & & & & Folfiri/Bev & 12 & 5,3 & 10,3 & 25,0 & $\mathrm{AE}(11.0)$ & \multicolumn{2}{|c|}{ "Other reasons" (6.7) } \\
\hline
\end{tabular}

PFS: Progression-Free Survival; OS: Overall Survival; Folfox: association of fluoropyrimidines and oxaliplatin; Folfiri: association of fluoropyrimidines and irinotecan; Cet: Cetuximab; Iri: irinotecan; Oxa: oxaliplatin; Bev: Bevacizumab; FU: fluorouracile; Pan: Panitumumab; Ced: Cediranib.

NR: Not reported; PD: Progressive Disease; AE: Adverse Events: PR: Patient Request.

Table 1: Descriptive analysis of selected studies.
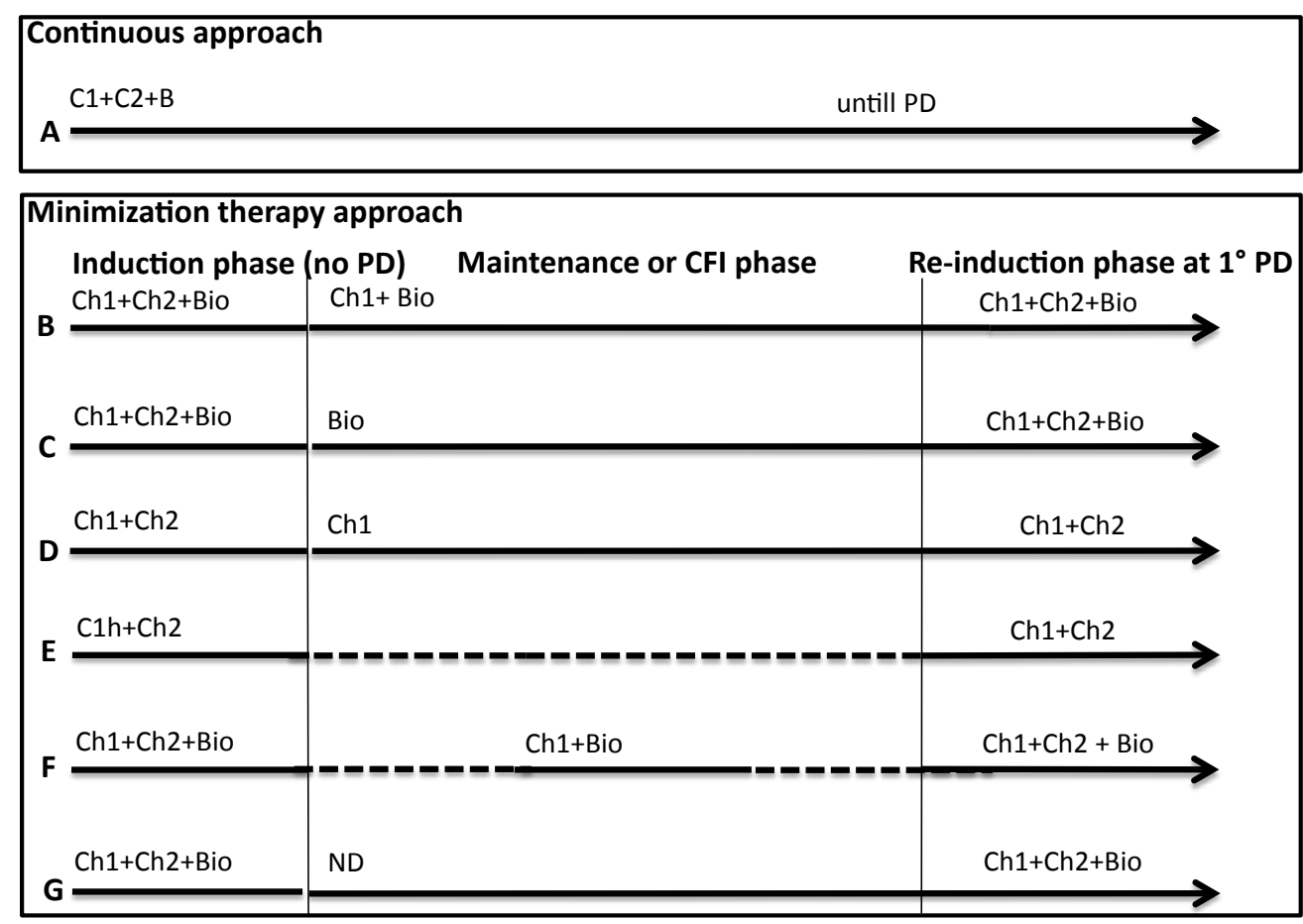

- Chemotherapy

$\mathrm{Ch}$ : chemotherapy; Ch1: favourable toxicity profile drug; $\mathrm{Ch} 2$ : unfavourable profile (i.e. cumulative toxicity or specific toxicity); PD: progressive disease.

Bio: biologic drug of previuos schedule; ND: new drug

Figure 1: Common treatment strategy in $\mathrm{mCRC}$.

studies. Notably, in phase III studies, from $11.0 \%$ to $45.0 \%$ of patients discontinue treatment for toxicity or their request independently from the efficacy. PR was the third cause of discontinuation ranging from $4.6 \%$ to $26.0 \%$ of patients and in some studies, it overcame the AErelated withdrawals (Table 1): this could be related to the desire of patients to have chemotherapy-free intervals.

\section{Discussion}

The most common treatment strategy in mCRC is the continuous administration of chemotherapy untill disease progression on intolerabe toxicity (Figure 1, line A). Therefore, from diagnosis onwards, an individual might spend most of his or her remaining life receiving continuous antitumor therapy, with the associated toxic effects, clinic visits, detriment to quality of life, and expense. The objective of the present survey was the description of real chemotherapy duration in phase III clinical trials of mCRC. No attempts were made to do metaanalysis because of treatments heterogeneity and strict selection of studies; furthermore, we cannot definitively rule out any relation between efficacy of treatments and time on therapy. However, duration of chemotherapy was quite homogeneous in first line studies reporting results with different chempotherapy schedules.

Why the patients discontinue treatments? There were no background data on factors predicting protocol adherence in phase III studies. It was reasonable to hypothesize that toxicity or progressions could account for the majority of withdrawals from a trial. However, in the present survey we found that PR (patient request) was the third cause of treatment discontinuation ranging from $4,6 \%$ to $26,0 \%$ of patients. Surprisingly, this phenomenon in some trials overcame the AE-related withdrawals. Thus, many patients treated with continuous approach chemotherapy, without progression of neither tumor nor serious adverse events; decide to stop the treatment and to remain in follow-up. Interestingly, in $\mathrm{mCRC}$, patient request as a reason of discontinuation therapy may hide the occurrence of particular toxicities that may negatively impact on physical, emotional, and social aspects of quality of life of patients independently from their severity (skin toxicity with anti-EGFR agents, asthenia or diarrhea with irinotecan and/or fluoropirimidines, neuropathy with oxaliplatin). 
Citation: Ottaiano A, Capuozzo M, Nasti G, Nappi A, De Divitiis C (2015) Duration of Chemotherapies in Phase III Studies on Metastatic Colorectal Cancer Treated With a Continuous Approach: A Seven-Year Survey. J Cancer Sci Ther 7: 202-205. doi:10.4172/1948-5956.1000351

Another detrimental effect of continuous therapy is psychological asthenia, a well-defined entity difficult to treat [5-7]. This aspect could be partially improved by managing patients in multidisciplinary teams involving psychologists. Other causes for study discontinuation could depend on logistics (transportation and/or mobilization difficulties, etc.) or personal/social reasons (loss of care-givers, change of opinion on protocol, etc.). Interestingly, in a series of $243 \mathrm{mCRC}$ patients treated at our Institution with capecitabine, oxaliplatin and bevacizumab from 2008 to 2014 (median number of cycle: 7, median duration: 6,2 months) psychological asthenia due to continuous therapy (23 patients) was the first cause among patient requests of therapy discontinuation (article in press).

Notably, it is difficult to identify the balance between advantages of prolonged tretaments and drawbacks due to patients refusal of continue exposure to chemotherapy; in fact, in the larg part of phase III trials, patients who discontinue treatments are excluded from final outcome analyses and the real causes behind "patient request" are not analyzed. However, our data may offer a critical reflection: the proportion of patient discontinuing treatments in phase III studies of mCRC for their request is surprisingly too high. For this reason, in recent years, oncologistis have hypothesized different approaches to minimize time on therapy and alternative strategies have been explored (OPTIMOX1 -Figure 1, line D-, OPTIMOX2 and COIN studies -Figure 1, line E) [8-10] in order to reduce side-effects and improve quality of life and adherence to protocols. How to minimize time on chemotherapy with palliative intent without compromising efficacy (many examples of intermittent strategies are shown in Figure $1)$ ? The question is completely open and should be explored in future phase III clinical trials ad hoc designed. Furthermore, causes of PR for therapy discontinuation should be explored and analyzed to reduce the proportion of withdrawals in phase III studies.

\section{References}

1. Heinemann $V$, von Weikersthal LF, Decker T, Kiani A, Vehling-Kaiser U, et al (2014) FOLFIRI plus cetuximab versus FOLFIRI plus bevacizumab as first-line treatment for patients with metastatic colorectal cancer (FIRE-3): a randomised, open-label, phase 3 trial. Lancet Oncol 15: 1065-1075

2. De Santis CE, Lin CC, Mariotto AB, Siegel RL, Stein KD, et al. (2014) Cancer treatment and survivorship statistics, 2014. CA Cancer J Clin 64: 252-271.

3. Brenner H, Kloor M, Pox CP (2014) Colorectal cancer. Lancet 383: 1490-1502.

4. Péron J, Maillet D, Gan HK, Chen EX, You BJ (2013) Adherence to CONSORT adverse event reporting guidelines in randomized clinical trials evaluating systemic cancer therapy: a systematic review. J Clin Oncol 31: 3957-3963.

5. Tonini G, Imperatori M, Vincenzi B, Frezza AM, Santini D (2013) Rechallenge therapy and treatment holiday: different strategies in management of metastatic colorectal cancer. J Exp Clin Cancer Res 32: 92.

6. Del Mastro L, Costantini M, Morasso G, Bonci F, Bergaglio M, et al (2002) Impact of two different dose-intensity chemotherapy regimens on psychological distress in early breast cancer patients. Eur J Cancer 38 359-366.

7. Morant R, Stiefel F, Berchtold W, Radziwill A, Riesen W (1993) Preliminary results of a study assessing asthenia and related psychological and biological phenomena in patients with advanced cancer. Support Care Cancer 1: 101-107.

8. Tournigand C, Cervantes A, Figer A, Lledo G, Flesch M, et al. (2006) OPTIMOX1: a randomized study of FOLFOX4 or FOLFOX7 with oxaliplatin in a stop-and-Go fashion in advanced colorectal cancer--a GERCOR study. J Clin Oncol 24: 394-400.

9. Adams RA, Meade AM, Seymour MT, Wilson RH, Madi A, et al. (2011) Intermittent versus continuous oxaliplatin and fluoropyrimidine combination chemotherapy for first-line treatment of advanced colorectal cancer: results of the randomised phase 3 MRC COIN trial. Lancet Oncol 12: 642-653.

10. Chibaudel B, Maindrault-Goebel F, Lledo G, Mineur L, André T, et al. (2009) Can chemotherapy be discontinued in unresectable metastatic colorecta cancer? The GERCOR OPTIMOX2 Study. J Clin Oncol 27: 5727-5733. 